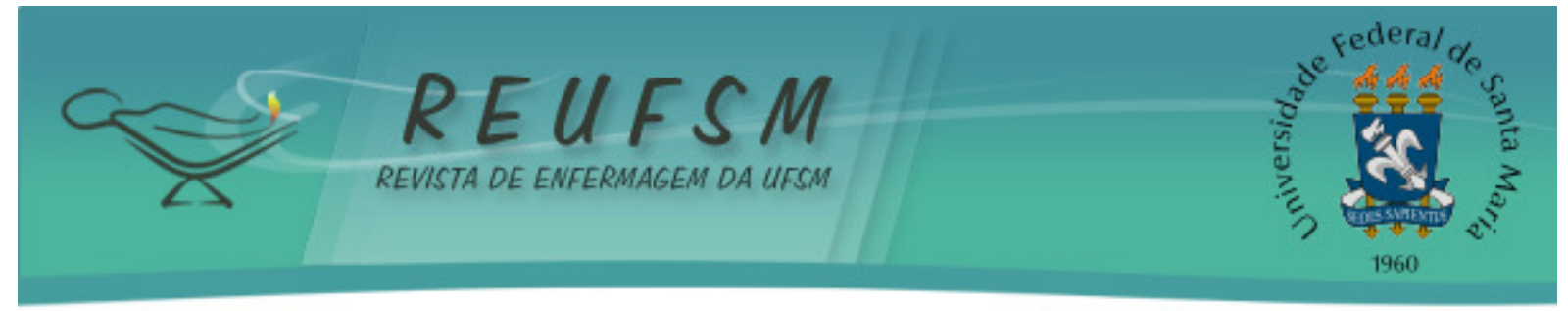

ARTIGO ORIGINAL

\title{
PERFIL EPIDEMIOLÓGICO DE CRIANÇAS E ADOLESCENTES COM SIDA: SÉRIE HISTÓRICA DE 1985 A 2012
}
EPIDEMIOLOGICAL PROFILE OF CHILDREN AND TEENS WITH AIDS: HISTORICAL SERIES OF 1985 TO 2012

\section{PERFIL EPIDEMIOLÓGICO DE LOS NIÑOS Y ADOLESCENTES CON SIDA: SÉRIE HISTÓRICA DE 1985 - 2012}

Márcio Cristiano de Melo ${ }^{1}$

\section{Doi: $10.5902 / 217976928956$}

RESUMO: Objetivo: descrever o perfil epidemiológico de crianças e adolescentes com SIDA em Porto Alegre-RS. Método: trata-se de um estudo epidemiológico e descritivo com dados do Sistema de Informação de Agravos de Notificação, disponibilizados pelo Sistema Único de Saúde no período de 1985 a 2012. Resultados: foram notificados 1.300 casos, sendo 1.221 crianças e 79 adolescentes, 662 masculinos e 638 femininos. 0 maior número de casos notificados foi observado em crianças menores de um ano $(n=470)$, o tipo de causa que foi responsável por $87,2 \%$ das notificações foi a transmissão vertical afetando com maior prevalência crianças menores de um ano a quatro anos. Conclusão: o perfil epidemiológico da SIDA para faixa etária de crianças menores de um ano a 14 anos caracterizou-se por uma redução gradual dos casos notificados a partir de 2003, em crianças e adolescentes do sexo masculino, brancas infectadas pelo vírus HIV por transmissão vertical.

Descritores: Síndrome de Imunodeficiência Adquirida; Notificação de Doenças; Sistemas de Informação; Criança; Adolescente

ABSTRACT: Aim: to describe the epidemiological profile of children and adolescents with AIDS in Porto Alegre-RS. Methods: it is a descriptive epidemiological study with data from the information system of reportable diseases, provided by the unified Health System in the period of 1985 to 2012. Results: 1,300 cases have been reported, 1,221 children and 79 adolescents, 662 male and 638 female. The largest number of reported cases was observed in children under one year $(n=470)$, the type of cause that accounted for $87.2 \%$ of the notifications was the mother-to-child transmission affecting with higher prevalence children under one year to four years. Conclusion: the epidemiological profile of AIDS for age group of children under one year to 14 years was characterized by a gradual reduction of reported cases from 2003, in children and adolescents white male HIV infected by mother-to-child transmission.

Descriptors: Acquired immunodeficiency syndrome; Disease notification; Information systems; Child; Adolescent

RESUMEN: Objetivo: describir el perfil epidemiológico de los niños y adolescentes con SIDA en Porto Alegre-RS. Metodología: se trata de un estudio epidemiológico descriptivo con los datos del sistema de información de enfermedades de notificación obligatoria, proporcionado por el sistema unificado de salud en el período de 1985 a 2012. Resultados: 1.300 casos fueron registrados, 1.221 niños y 79 adolescentes, 662 masculinos y 638 femeninos. El mayor número de casos se observó en niños menores de un año $(n=$ 470), el tipo de causa que representaron $87.2 \%$ de las notificaciones era la transmisión

\footnotetext{
${ }^{1}$ Enfermeiro Especialista em Saúde Coletiva pele Escola de Enfermagem da Universidade Federal de Minas Gerais, Docente de Apoio Pedagógico da Escola de Saúde Pública de Minas Gerais e Docente do Curso de ACS do PRONATEC pela UFV - Campus Florestal. Belo Horizonte, MG, Brasil. E-mail: enf.marciomelo@gmail.com
} 


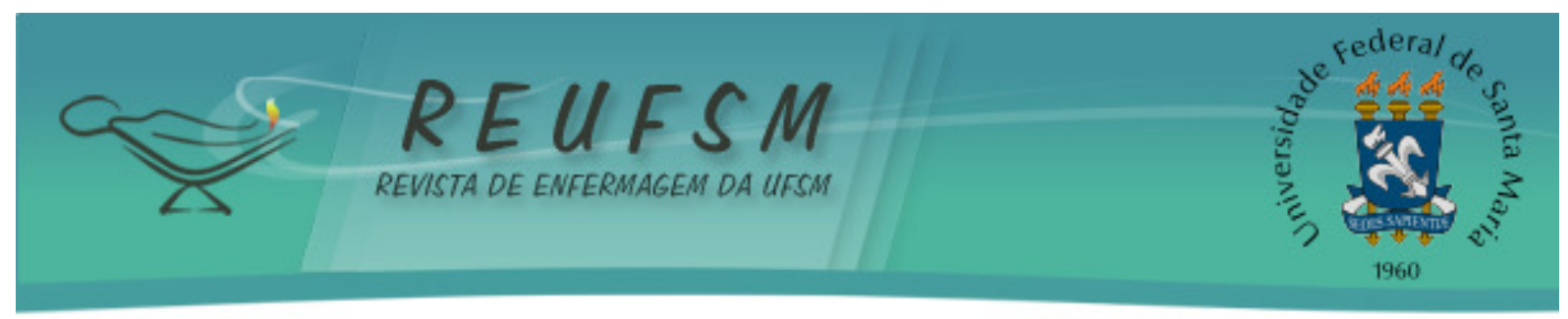

vertical, afectando con mayor prevalência niños menores de un año a cuatro años. Conclusión: el perfil epidemiológico del SIDA por grupo de edad de los niños menores de un año a 14 años se caracterizó por una reducción gradual de los casos divulgados de 2003, en niños y adolescentes infectado por HIV hombre blanco por transmisión.

Descriptores: Síndrome de inmunodeficiencia adquirida; Notificación de enfermedad; Sistemas de información; Niño; Adolescente

\section{INTRODUÇÃO}

O surgimento da infecção pelo HIV em crianças está relacionado à expansão da epidemia entre mulheres. No Brasil começaram a aparecer os primeiros casos de transmissão do HIV para bebês, com isso, a frequência de óbitos decorrentes da infecção nas crianças, e também nos adultos era elevada, frente à inexistência de medicamentos ou procedimentos que impedissem a transmissão vertical. Somente em 1996 medidas profiláticas em relação à transmissão vertical foram desenvolvidas pela medicina. ${ }^{1}$

No Brasil, o Estatuto da Criança e do Adolescente (ECA), Lei 8.069, de 1990, considera criança a pessoa até 12 anos de idade incompletos e define a adolescência como a faixa etária de 12 a 18 anos de idade (artigo $2^{\circ}$ ), e, em casos excepcionais e quando disposto na lei, o estatuto é aplicável até os 21 anos de idade (artigos 121 e 142). ${ }^{2}$

A adolescência é uma etapa da vida onde ocorrem muitas transformações biológicas, psíquicas e sociais. Dentre estas, o comportamento sexual do adolescente é um ponto normal do desenvolvimento ${ }^{3}$ e, quando se dá início à atividade sexual, aumenta a vulnerabilidade às doenças sexualmente transmissíveis (DST's) e à SIDA ${ }^{4}$. Dentre os fatores que podem colocar os adolescentes em maior risco para as DST's estão: a idade precoce de início da atividade sexual, uso incorreto ou inconsistente de preservativos e experimentação com álcool e outras drogas. ${ }^{5}$

Em pesquisa feita com 2.485 jovens, demonstrou-se que apenas $51,7 \%$ tinham conhecimento correto das formas de transmissão da SIDA, e, em torno de $97 \%$ sabem que podem ser infectados nas relações sexuais sem uso de preservativo. ${ }^{4}$

O vírus da Imunodeficiência Humana (HIV) é pertencente à classe dos retrovírus Lentiviridae causador da Síndrome da Imunodeficiência Adquirida (SIDA). ${ }^{6}$ A transmissão do HIV pode ocorrer por meio de quatro vias: sexual, sanguínea, parenteral, além da transmissão ocupacional, na qual ocorre o contato e/ou troca de sangue ou de secreção orgânica que contém o vírus ou células parasitadas pelo mesmo. ${ }^{7-8}$

Os infectados pelo HIV evoluem para grave disfunção do sistema imunológico, à medida que vão sendo destruídos os linfócitos T CD4+, uma das principais células-alvo do vírus. A contagem de linfócitos T CD4+ é importante marcador dessa imunodeficiência, sendo utilizado tanto na avaliação do tratamento e prognóstico quanto em uma das definições de caso de SIDA. ${ }^{8}$

Atualmente, apenas as pessoas que desenvolveram a SIDA, gestantes com HIV e crianças expostas ao vírus são notificadas junto aos sistemas de vigilância do Ministério da Saúde. A ampliação de notificação dos casos de diagnóstico positivo para o HIV a partir de 2013 será apresentada a representantes dos movimentos sociais antes de ser implementada. Se aprovada, tal medida aprimorará a resposta à SIDA, permitirá iniciar o tratamento em tempo oportuno, visando, com isso, que a doença não se manifeste. A notificação irá contribuir para precisar os dados e redirecionar a vigilância epidemiológica. ${ }^{9}$

Ocorreu no Brasil um importante deslocamento no perfil da SIDA, incluindo cada vez mais mulheres de 15-40 anos em plena idade reprodutiva. Essa heterossexualização e feminização da epidemia têm numerosas consequências, dentre elas, o aumento do número de crianças infectadas pelo HIV tendo a transmissão vertical como principal via de infecção. ${ }^{10}$

O primeiro caso de SIDA notificado no Rio Grande do Sul foi em 1983, sendo que até junho de 2010, o estado notificou 46.001 casos no Sistema de Informação de Agravos 


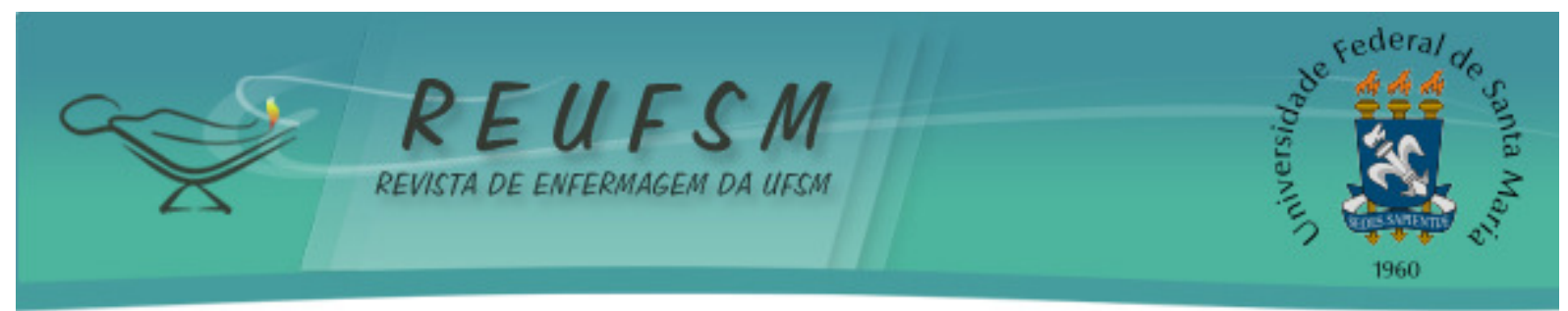

de Notificação (SINAN). Em 2009, a taxa de incidência do estado foi de 47,5/100.000 habitantes, a da região sul, 32,4 e a do Brasil, 20,1. De 1997 a junho de 2010, foram notificados 1.375 casos de SIDA em menores de cinco anos. ${ }^{11}$

Os cinco municípios que apresentam o maior número de casos, até junho de 2010, foram: Porto Alegre (24.706), Canoas (2.252), São Leopoldo (2.102), Pelotas (1.863) e Viamão (1.825). Em relação à gestante HIV+, foram notificados 11.931 casos no Rio Grande do Sul, de 2000 a junho de 2010 e, 1.645 casos de SIDA por transmissão vertical até junho de 2010. ${ }^{11}$

Em meio à reflexão deste trabalho, surgiu o questionamento sobre estudos voltados à população foco desta pesquisa e o porquê de não haver estudos epidemiológicos específicos sobre casos notificados de SIDA em crianças e adolescentes residentes em Porto Alegre.

Neste trabalho, procurou-se descrever o perfil epidemiológico de crianças e adolescentes com SIDA em Porto Alegre-RS segundo sexo, idade, escolaridade e categoria de exposição.

\section{MÉTODO}

Trata-se de um estudo epidemiológico, descritivo de série histórica realizado no município de Porto Alegre pertencente ao estado de Rio Grande do Sul, com dados obtidos a partir do Sistema de Informação de Agravos de Notificação (SINAN), disponibilizados pelo Departamento de Informática do Sistema Único de Saúde. A escolha do período, 1985 a 2012, ocorreu pela disponibilidade desses dados no Departamento de Informática do Sistema Único de Saúde no momento da coleta, em maio de 2013.

Optou-se por trabalhar somente com os casos notificados caracterizados pelo desenvolvimento da SIDA ao invés de agregar, também, os casos de pessoas diagnosticadas com HIV, o que demandaria a necessidade de recorrer à Secretaria Municipal da cidade para a coleta de tais dados, inviabilizando a proposta da pesquisa de utilizar como fonte de coleta o Departamento de Informática do Sistema Único de Saúde.

0 estudo foi realizado com base nos casos de SIDA notificados ao SINAN do município de Porto Alegre. Juntamente com as faixas etárias propostas nesta pesquisa $(0 \mathrm{a}$ 14 anos), foram analisadas as seguintes variáveis constantes da ficha de notificação disponibilizada pelo Departamento de DST, SIDA e Hepatites Virais do Ministério da Saúde (CID-10 B-24 e Z-21): sexo, raça/cor (branca, preta, amarela, parda, indígena, ignorada), escolaridade em anos de instrução (nenhum e ignorado) e categoria de exposição (heterossexual, homossexual, bissexual, usuário de drogas injetáveis, hemofílico, transfusão, transmissão vertical, ignorado).

Porto Alegre possui uma extensão territorial de $496.682 \mathrm{Km}^{2}$ e uma população estimada no ano de 2012 de 1.416 .714 habitantes. ${ }^{12}$

A notificação é feita pelo preenchimento e envio da Ficha de Investigação Epidemiológica de Caso de SIDA, adulto ou criança, disponível no SINAN, que deve ser preenchida pelo médico ou outro profissional de saúde capacitado para tal, no exercício de sua função. A Portaria $\mathrm{n}^{\circ} 2.325 / \mathrm{GM}$, de 08 de dezembro de 2003, regulamenta a notificação de doenças compulsórias em todo o país, inclusive da SIDA. ${ }^{6}$

Todas as categorias das variáveis foram escolhidas conforme são apresentadas no SINAN, não havendo readequação das mesmas pelos autores de acordo com os objetivos do estudo.

Como critério de inclusão ao estudo utilizou-se a faixa etária para a seleção dos dados disponibilizados no SINAN, e, excluindo assim, os casos que não foram notificados.

A coleta dos dados procedeu-se pelo Departamento de Informática do Sistema Único de Saúde, os dados foram apresentados em forma de tabelas e gráficos de distribuição de frequências e de coeficientes de incidência de SIDA para cada 100.000 habitantes, construídos com o auxílio do software Excel 2007 para Windows Vista e o 


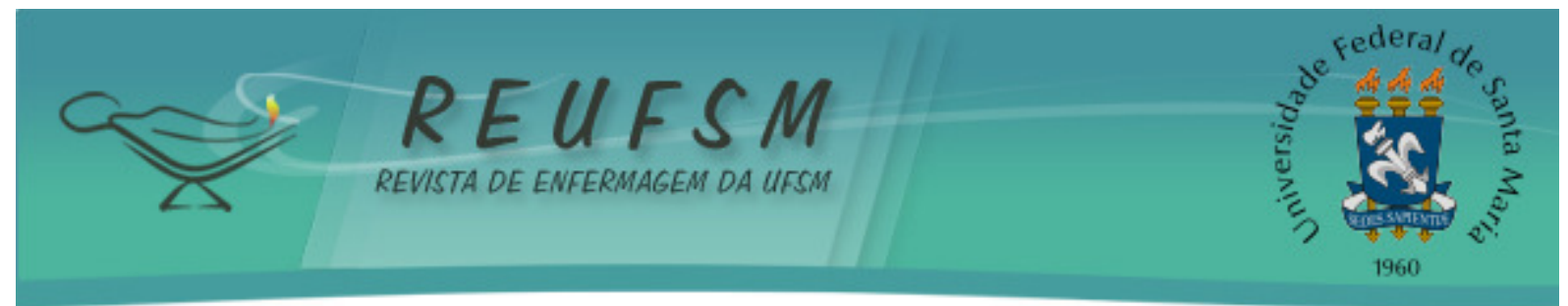

programa de tabulação de dados TabNet no Ministério da Saúde. Os coeficientes de incidência definem-se como medidas por excelência do risco da doença e do agravo. Os valores calculados seguiram como base as fórmulas recomendadas para estudos epidemiológicos ${ }^{13}$, de acordo com a Figura 1.

$$
\text { Coeficiente de Incidência }=\quad \begin{gathered}
\begin{array}{c}
\mathrm{n}^{\circ} \text { de casos de uma nova doença, ocorridos em } \\
\text { determinada comunidade, em certo período de } \\
\text { tempo }
\end{array} \\
\cline { 2 - 2 } \begin{array}{c}
\mathrm{n}^{\circ} \text { de pessoas expostas ao risco de adquirir a } \\
\text { doença no referido período }
\end{array}
\end{gathered}
$$

Figura 1 - Fórmula para o cálculo do Coeficiente de Incidência

Para o cálculo de incidência anual dos casos de SIDA, foram usadas como denominadores as populações dos censos demográficos de 1986 a 2010, fornecidos pelo Departamento de Informática do Sistema Único de Saúde.

Não houve a necessidade de submeter o presente estudo ao Comitê de Ética de Pesquisas em Seres Humanos por basear-se em dados públicos e de livre acesso.

\section{RESULTADOS}

O número de casos de SIDA notificados, no período de 1985 a 2012, na cidade de Porto Alegre incluiu 1.300 crianças, sendo 1.221 crianças ( 0 a 12 anos incompletos), das quais 621 eram do sexo masculino e 600 eram do sexo feminino e 79 adolescentes (12 a 14 anos), dos quais 41 eram do sexo masculino e 38 eram do sexo feminino.

No que diz respeito à faixa etária, o maior número de casos foi observado em crianças menores de um ano $(n=470)$ representando $36,2 \%$ do total, sendo 255 do sexo masculino e 215 do sexo feminino. Com relação ao grau de escolaridade da população em estudo, observou-se que não há registro de tal informação, todos os casos foram notificados como: nenhum ano de estudo $(n=755)$ ou ignorado $(n=545)$ (Tabela 1$)$.

Com relação à raça/cor verificou-se que os brancos apresentam maior incidência da doença $(n=279), 68,5 \%$ dos casos $(n=890)$, relacionados à raça/cor, foram notificados como ignorados, não permitindo que tal dado possa ser usado para definição de um perfil epidemiológico.

No que diz respeito à categoria de exposição, $87,2 \%$ dos casos $(n=1.134)$ foram por transmissão vertical (ocorre pela passagem do vírus da mãe para o bebê durante a gestação, o trabalho de parto, o parto propriamente dito - contato com as secreções cérvico-vaginais e sangue materno, ou a amamentação) ${ }^{14}$ sendo 567 casos masculinos $(85,6 \%)$ e 567 casos femininos $(88,9 \%)$, mesmo apresentando o mesmo número de casos entre os sexos, percebe-se que entre as mulheres a proporção por transmissão vertical foi maior (Tabela 1). 


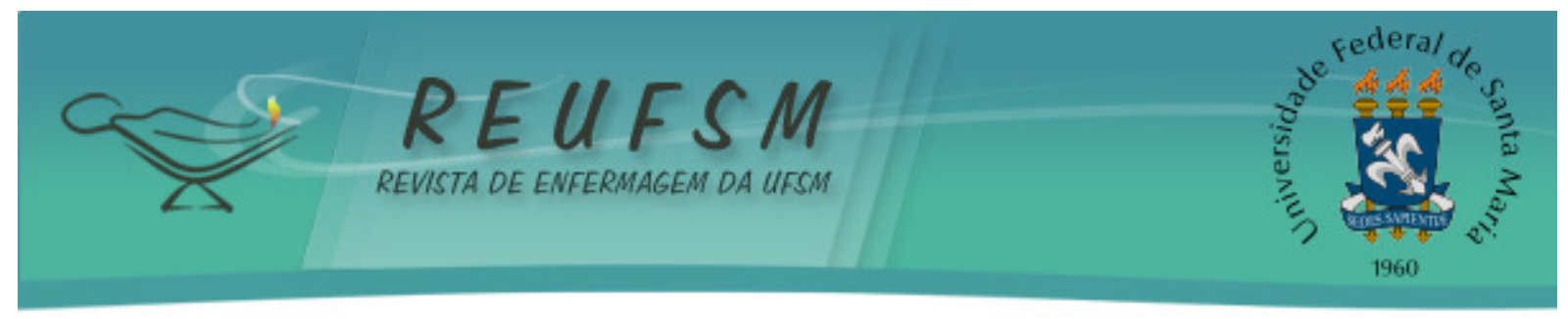

Tabela1 - Casos de SIDA em indivíduos, segundo variáveis sociodemográficas e categoria de exposição. Porto Alegre - RS, 1985 a 2012.

\begin{tabular}{|c|c|c|c|c|c|c|}
\hline \multirow[b]{2}{*}{ Variáveis } & \multicolumn{2}{|c|}{ Masculino $(n=662)$} & \multicolumn{2}{|c|}{ Feminino $(n=638)$} & \multicolumn{2}{|c|}{ Total $(n=1.300)$} \\
\hline & $n$ & $\%$ & $\mathrm{n}$ & $\%$ & $\mathrm{n}$ & $\%$ \\
\hline \multicolumn{7}{|l|}{ Faixa etária (anos) } \\
\hline Menor de 1 & 255 & 38.5 & 215 & 33.7 & 470 & 36.2 \\
\hline 01 a 04 & 198 & 29.9 & 224 & 35.1 & 2 & 32.5 \\
\hline 05 a 09 & 132 & 19.9 & 1 & 20.1 & 260 & 20 \\
\hline 10 a 14 & 77 & 11.6 & 71 & 11.1 & 148 & 11.4 \\
\hline \multicolumn{7}{|l|}{ Anos de estudo } \\
\hline Nenhum & 391 & 59.1 & 364 & 57.1 & 755 & 58.1 \\
\hline Ignorado & 271 & 40.9 & 274 & 42.9 & 545 & 41.9 \\
\hline \multicolumn{7}{|l|}{ Raça/cor } \\
\hline Branca & 135 & 20.4 & 144 & 22.6 & 279 & 21.5 \\
\hline Preta & 37 & 5.6 & 41 & 6.4 & 78 & 6.0 \\
\hline Parda & 28 & 4.2 & 24 & 3.8 & 52 & 4.0 \\
\hline Indígena & 1 & 0.2 & 2. & - & 1 & 0.1 \\
\hline Ignorado & 461 & 69.6 & 429 & 67.2 & 890 & 68.5 \\
\hline \multicolumn{7}{|l|}{ Categoria de Exposição } \\
\hline Heterossexual & 2 & 0.3 & 59 & 9.2 & 61 & 4.7 \\
\hline Homossexual & 2 & 0.3 & - & - & 2 & 0.2 \\
\hline Bissexual & 1 & 0.2 & - & - & 1 & 0.1 \\
\hline UDI & 10 & 1.5 & 3 & 0.5 & 13 & 1.0 \\
\hline Hemofílico & 7 & 1.1 & - & - & 7 & 0.5 \\
\hline Transfusão & 2 & 0.3 & 1 & 0.2 & 3 & 0.2 \\
\hline Transmissão Vertical & 567 & 85.6 & 567 & 88.9 & 1134 & 87.2 \\
\hline Ignorado & 71 & 10.7 & 8 & 1.3 & 79 & 6.1 \\
\hline
\end{tabular}

Fonte: Sistema de Informação de Agravos de Notificação, DATASUS, 2013.

No período estudado, ao detalhar a idade, percebemos que a maior incidência de novos casos notificados acontece em crianças com menos de um ano até dois anos de idade (56,9\% dos casos notificados), conforme apresentado no Gráfico 1. 

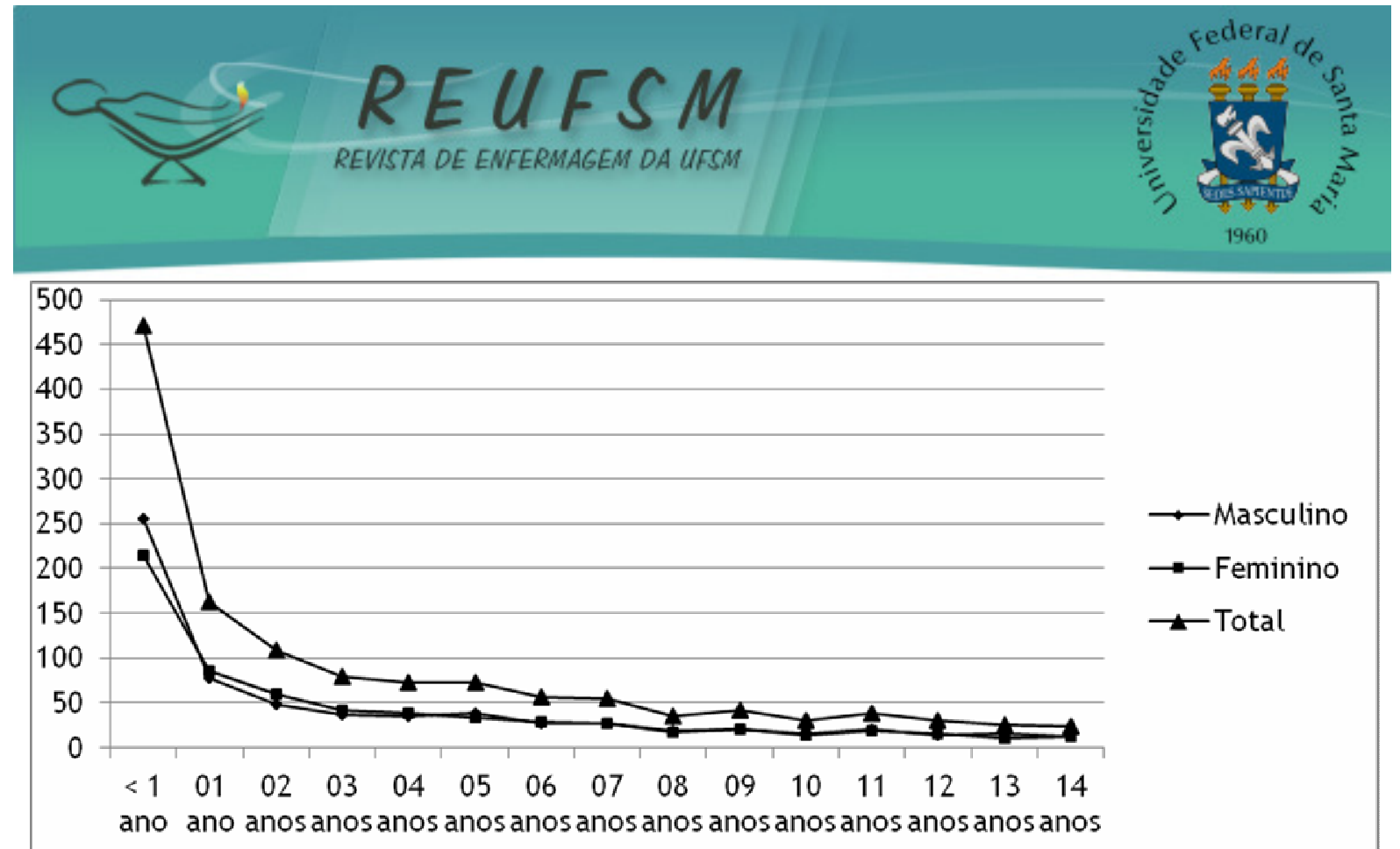

Gráfico 1 - Frequência de novos casos segundo idade detalhada. Porto Alegre - RS, 1985 a 2012.

Ao analisar o total de casos notificados entre as faixas etárias estudas, no estado do Rio Grande do Sul constatamos que Porto Alegre detém, no início da notificação, os primeiros casos de SIDA, e, que a curva de total de casos no estado acompanha a curva de total de casos da capital, como demonstrado no Gráfico 2.

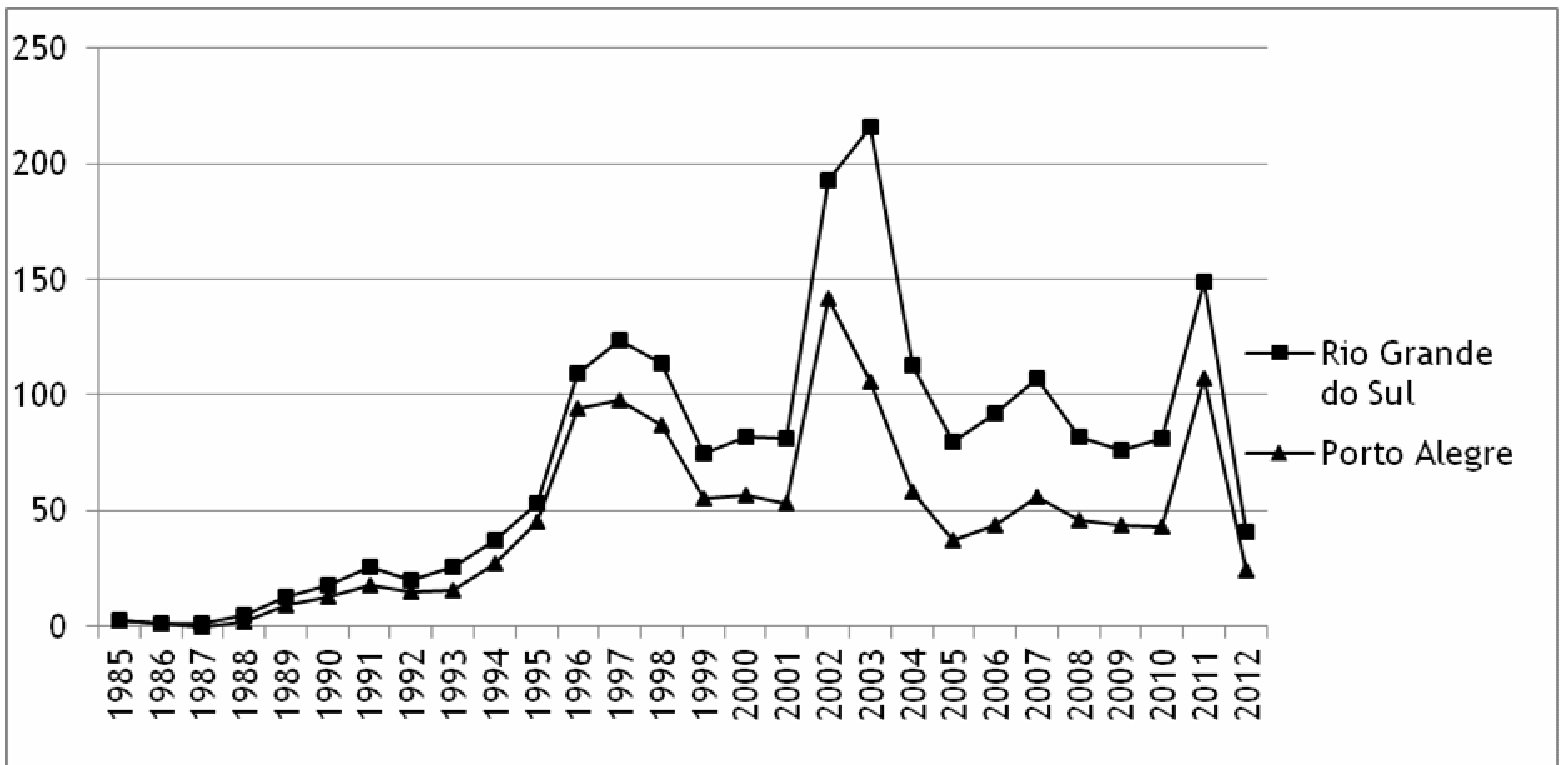

Gráfico 2 - Prevalência total de casos segundo estado e sua respectiva capital. Porto Alegre - RS, 1985 a 2012.

Entre os sexos houve um aumento da prevalência a partir de 1994 (3,5/100.000 habitantes) alcançando seu maior índice no ano de 1996 (9,5/100.000 habitantes) para o sexo masculino e em 1997 (6,9/100.000 habitantes) para o sexo feminino. A prevalência de casos masculinos teve uma queda considerável no ano de 2003 (3,5/100.000 habitantes) com um registro 1,3 maior em 2007 (4,6/100.000 habitantes) e a partir desse ano a prevalência tendeu a diminuir até 2012 (0,9/100.000 habitantes). Para o sexo feminino houve queda, também, no ano de 2003 (4,3/100.000 habitantes) onde passou a manter média de 3,6/100.000 habitantes até 2011 e caiu para 0,7/100.00 habitantes em 2012 (Tabela 2). 


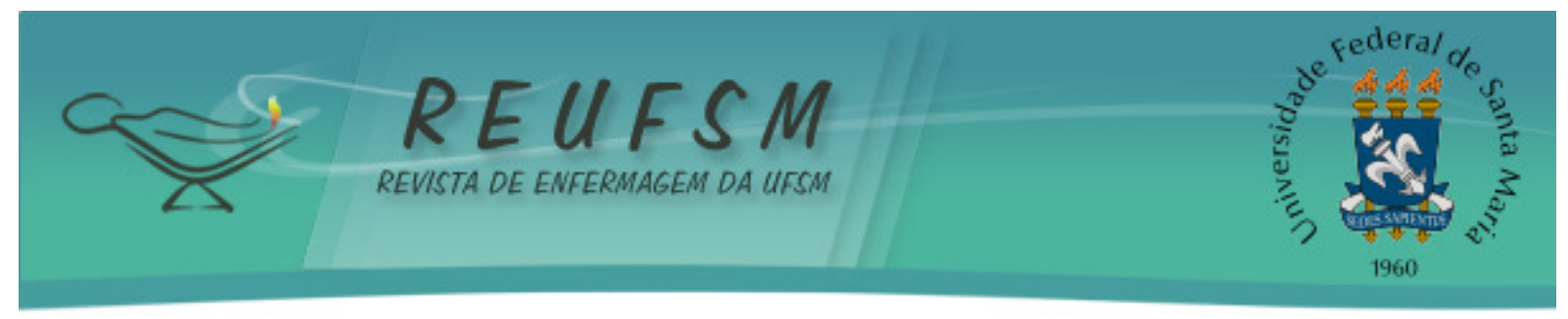

Tabela 2 - Coeficiente de incidência da SIDA (por 100.000 habitantes), segundo o ano de notificação e sexo. Porto Alegre - RS, 1985 a 2012.

\begin{tabular}{|c|c|c|c|c|c|c|c|c|c|}
\hline \multirow[b]{2}{*}{ Anos } & \multirow[b]{2}{*}{ Mas } & \multicolumn{2}{|l|}{ Pop Res } & \multirow[b]{2}{*}{ Fem } & \multirow[b]{2}{*}{ Pop Res Fem } & \multirow[b]{2}{*}{$\mathrm{Cl}$ Fem } & \multicolumn{3}{|c|}{ Pop Res } \\
\hline & & Mas & $\mathrm{Cl}$ Mas & & & & Total & Total & $\mathrm{Cl}$ Total \\
\hline 1985 & 3 & 556385 & 0.5 & - & 631738 & - & 3 & 1188123 & 0.3 \\
\hline 1986 & - & 562044 & - & 1 & 638917 & 0.2 & 1 & 1200961 & 0.1 \\
\hline 1988 & - & 573077 & - & 2 & 652932 & 0.3 & 2 & 1226009 & 0.2 \\
\hline 1989 & 9 & 578389 & 1.6 & 2 & 659675 & 0.3 & 11 & 1238064 & 0.9 \\
\hline 1990 & 11 & 583579 & 1.9 & 4 & 666266 & 0.6 & 15 & 1249845 & 1.2 \\
\hline 1991 & 12 & 589555 & 2.0 & 8 & 673848 & 1.2 & 20 & 1263403 & 1.6 \\
\hline 1992 & 13 & 588828 & 2.2 & 5 & 669795 & 0.7 & 18 & 1258623 & 1.4 \\
\hline 1993 & 10 & 597351 & 1.7 & 11 & 682763 & 1.6 & 21 & 1280114 & 1.6 \\
\hline 1994 & 21 & 604072 & 3.5 & 24 & 690434 & 3.5 & 45 & 1294506 & 3.5 \\
\hline 1995 & 28 & 610617 & 4.6 & 30 & 697921 & 4.3 & 58 & 1308538 & 4.4 \\
\hline 1996 & 57 & 602982 & 9.5 & 45 & 685897 & 6.6 & 102 & 1288879 & 7.9 \\
\hline 1997 & 47 & 607299 & 7.7 & 48 & 690809 & 6.9 & 95 & 1298108 & 7.3 \\
\hline 1998 & 56 & 610929 & 9.2 & 40 & 694940 & 5.8 & 96 & 1305869 & 7.4 \\
\hline 1999 & 39 & 614748 & 6.3 & 31 & 699285 & 4.4 & 70 & 1314033 & 5.3 \\
\hline 2000 & 43 & 635820 & 6.8 & 37 & 724770 & 5.1 & 80 & 1360590 & 5.9 \\
\hline 2001 & 50 & 641760 & 7.8 & 48 & 731552 & 6.6 & 98 & 1373312 & 7.1 \\
\hline 2002 & 53 & 646508 & 8.2 & 50 & 736946 & 6.8 & 103 & 1383454 & 7.4 \\
\hline 2003 & 23 & 651467 & 3.5 & 32 & 742620 & 4.3 & 55 & 1394087 & 3.9 \\
\hline 2004 & 28 & 656412 & 4.3 & 22 & 748258 & 2.9 & 50 & 1404670 & 3.6 \\
\hline 2005 & 22 & 667639 & 3.3 & 30 & 761055 & 3.9 & 52 & 1428694 & 3.6 \\
\hline 2006 & 26 & 673367 & 3.9 & 28 & 767573 & 3.6 & 54 & 1440940 & 3.7 \\
\hline 2007 & 31 & 679066 & 4.6 & 29 & 774009 & 3.7 & 60 & 1453075 & 4.1 \\
\hline 2008 & 22 & 668256 & 3.3 & 32 & 761964 & 4.2 & 54 & 1430220 & 3.8 \\
\hline 2009 & 19 & 670856 & 2.8 & 28 & 765268 & 3.7 & 47 & 1436124 & 3.3 \\
\hline 2010 & 18 & 653787 & 2.8 & 23 & 755564 & 3.0 & 41 & 1409351 & 2.9 \\
\hline 2011 & 14 & 655523 & 2.1 & 23 & 757571 & 3.0 & 37 & 1413094 & 2.6 \\
\hline 2012 & 6 & 657201 & 0.9 & 5 & 759513 & 0.7 & 11 & 1416714 & 0.8 \\
\hline
\end{tabular}

Fonte: Estimativas populacionais enviadas para o TCU, estratificadas por idade e sexo pelo MS/SGEP/DATASUS, 2013.

\section{DISCUSSÃO}

A epidemia de SIDA no Brasil é, de fato, o somatório de subepidemias microrregionais, em interação permanente, devido aos diferentes momentos de introdução do HIV no território nacional, às diferentes condições de vida das populações atingidas, às 


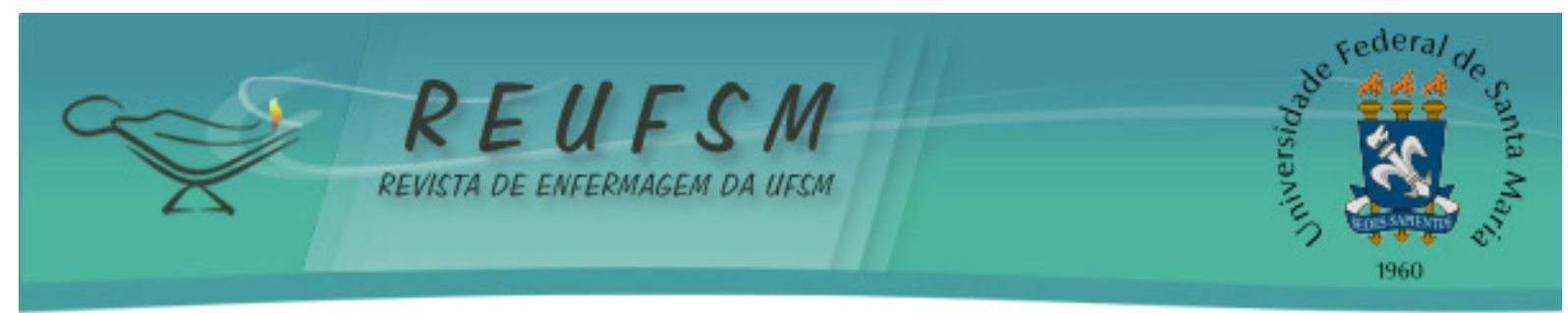

distintas composições das populações regionais, aos padrões de mobilidade da população e à diversidade de arranjos e padrões de comportamento sexual. Considerada como uma doença emergente representando um dos maiores problemas de saúde da atualidade em virtude de seu caráter pandêmico e gravidade. ${ }^{6}$

No presente estudo a incidência da SIDA foi maior nas idades onde a amamentação é exclusiva, predominante ou complementar, podendo assim, caracterizar aumento da prevalência de transmissão vertical na amamentação. ${ }^{15-16} \mathrm{~A}$ prática do aleitamento materno por mães soropositivas é contra indicada no Brasil. ${ }^{17} \mathrm{~A}$ taxa de transmissão vertical do HIV sem qualquer intervenção situa-se em torno de $20 \%$ e, com o uso combinado do Protocolo 076 do SIDA Clinical Trial Group (PACTG 076), ocorre uma redução de $67,5 \% .{ }^{18}$ No Brasil, a transmissão vertical diminuiu de $16,0 \%$ para 3,7\% entre 1997 e 2002, embora a implementação deste protocolo ainda não esteja plenamente concluída. ${ }^{19}$

Com relação ao grau de escolaridade, não há dados que possam demonstrar significância, mesmo sabendo que usualmente as crianças e adolescentes entre seis e 14 anos já frequentam o meio escolar.

Mesmo mantendo um alto índice de notificações com relação à variável "ignorado" $(68,5 \%)$, percebe-se que $21,5 \%$ dos casos estão presentes na raça branca seguida da raça preta (6\%), tal dado não foi encontrado em outros estudos para parâmetro de comparação. A não inserção da informação referente à raça/cor ou qualquer outra variável dificulta a realização de estudos epidemiológicos, tal apontamento é identificado em estudo semelhante com outras faixas etárias. ${ }^{20}$

Hoje sabemos que a transmissão vertical se tornou a principal via de infecção do HIV em crianças. ${ }^{8}$ Como analisado nesta pesquisa, tal afirmação se faz verdadeira. Este dado assemelha-se a pesquisas que abordam a temática da transmissão vertical. ${ }^{15-16,21-22}$

Para esses casos, torna-se evidente que políticas de saúde que envolva equipes multidisciplinares capacitadas para que haja acompanhamento e seguimento dessas crianças, garantindo melhores condições de vida. ${ }^{23-24}$

A prevalência de casos notificados de SIDA atualmente nas faixas etárias estudadas está abaixo de 1/100.000 habitantes devido a melhorias e avanços no tratamento, tanto medicamentoso como acompanhamento dos infectados. A resposta ao tratamento antirretroviral tem se mostrado mais satisfatória devido políticas de saúde melhores e mais eficazes. ${ }^{25-27}$

Conhecer a população e como a doença caracteriza-se nela faz com que haja melhorias no modo de traçar novos planos de cuidados e também para adotar políticas adequadas com relação à transmissão, diagnóstico e tratamento. ${ }^{18,26-27}$

\section{CONCLUSÃO}

O perfil epidemiológico da SIDA para faixa etária de crianças menores de um ano a 14 anos caracterizou-se por uma redução gradual dos casos notificados a partir do ano de 2003, predominando em crianças e adolescentes do sexo masculino, brancas e que se infectam pelo vírus HIV por transmissão vertical.

Como limitação para este estudo, percebemos que em quase todos os itens da ficha de notificação ocorreu um alto índice de marcação do item "ignorado", podendo estar relacionado a um preenchimento inadequado da mesma. Tais dados são ferramentas para estudos futuros e sua subnotificação impede o planejamento de políticas de saúde satisfatórias.

O grande número de casos onde a ausência de dados na ficha de notificação lança um alerta para que melhorias como: capacitação dos profissionais diretamente ligados à notificação sejam tomadas. Deve haver uma investigação sobre os indivíduos notificados e suas condições atuais de vida pra que planos de cuidado sejam traçados.

Estudos deste tipo permitem que as vigilâncias em saúde e epidemiológica, acompanhem a tendência temporal e espacial da doença, infecções e formas de contágio 


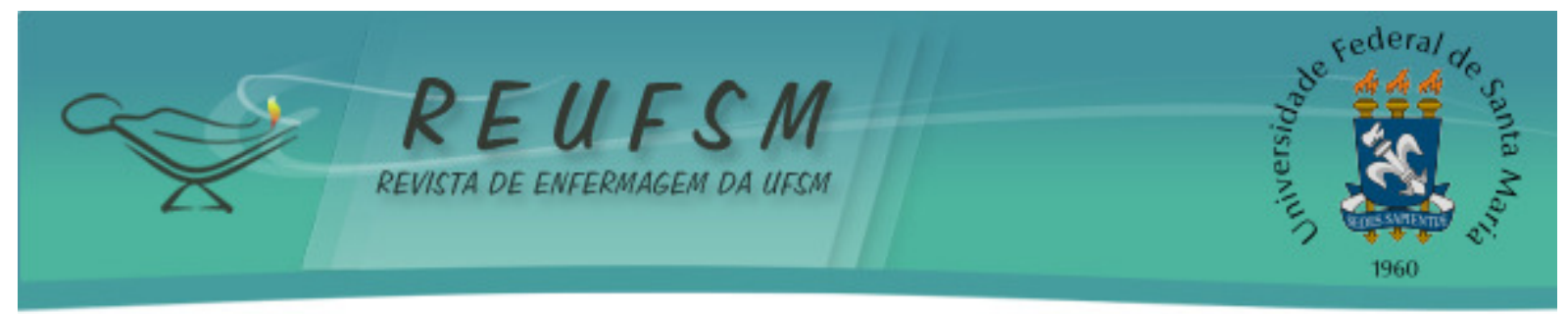

visando nortear suas ações, torna-se um poderoso componente objetivo para novas políticas de saúde voltadas às crianças e aos adolescentes, permitindo aos dirigentes municipais uma visão ampla e direcionada da situação real de saúde de sua população.

Há a necessidade de implantação de ações de promoção e manutenção da saúde e prevenção do adoecimento na população, e, para tanto é imprescindível aprimorar o acesso ao serviço, adotar estratégias de educação em saúde e de cuidado coerentes com cada fase da vida. Estudos como este contribuem para a promoção de novas estratégias de políticas públicas de saúde.

\section{REFERÊNCIAS}

1. Cunha CC. Revelando vozes, desvendando olhares: os significados do tratamento para o HIV/AIDS [dissertação]. Rio de Janeiro: Fundação Oswaldo Cruz, Escola Nacional de Saúde Pública; 2004.

2. Brasil. Lei n. 8.069, de 13 de julho de 1990. Estatuto da Criança e do Adolescente. Diário Oficial da União (texto retificado), Brasília, 27 set. 1990. Seção 1, p. 18551.

3. Machado DM, Succi RC, Turato ER. Transitioning adolescents living with HIV/AIDS to adult-oriented health care: an emerging challenge. J Pediatr (Rio J) [Internet]. 2010 Nov/Dec [acesso em 2013 June 18];86(6):465-72. Disponível em: http: //www.scielo.br/pdf/jped/v86n6/v86n6a04.pdf.

4. Brasil. Ministério da Saúde. Secretaria de Vigilância em Saúde. Departamento de DST, Aids e Hepatites Virais. $1^{a}$ ed. Recomendações para a atenção integral a adolescentes e jovens vivendo com HIV/Aids. Brasília (DF); 2013. p. 11.

5. Guerra CPP, Seidl EMF. Crianças e adolescentes com HIV/Aids: revisão de estudos sobre revelação do diagnóstico, adesão e estigma. Paideia [Internet]. 2009 jan/abr [acesso em 2013 jun 18];19(42):59-65. Disponível em: http: //www.scielo.br/pdf/paideia/v19n42/08.pdf.

6. Brasil. Ministério da Saúde. Secretaria de Vigilância em Saúde. Departamento de Vigilância Epidemiológica. Doenças infecciosas e parasitárias - guia de bolso. $8^{\mathrm{a}}$ ed. Brasília (DF); 2010. p. 80.

7. Brasil. Ministério da Saúde. Programa Nacional de DST/AIDS. Boletim Epidemiológico. Brasília (DF); 2008.

8. Brasil. Ministério da Saúde. Boletim Epidemiológico - AIDS/DST. Brasília (DF); 2010.

9. Brasil. Ministério da Saúde. Diagnóstico precoce: casos de HIV passarão a ser notificados no Brasil. Portal da Saúde [Internet]. 2012 dez 27 [acesso em 2013 maio 15]. Disponível em: http://portalsaude.saude.gov.br/portalsaude/noticia/8774/162/casos-de-hivpassarao-a-\%3Cbr\%3Eser-notificados-no-brasil.html.

10. Joint United Nation Programme on HIV/AIDS. AIDS epidemic update. Geneva: UNAIDS; 2006.

11. Brasil. Ministério da Saúde. Secretaria de Vigilância em Saúde. Sistema nacional de vigilância em saúde: relatório de situação: Rio Grande do Sul. Brasília (DF); 2011. (Série C; Projetos, Programas e Relatórios).

12. Instituto Brasileiro de Geografia e Estatísticas (IBGE). Estimativas da população residente com data de referência $1^{\circ}$ de julho de 2012. Rio de Janeiro; 2013. 


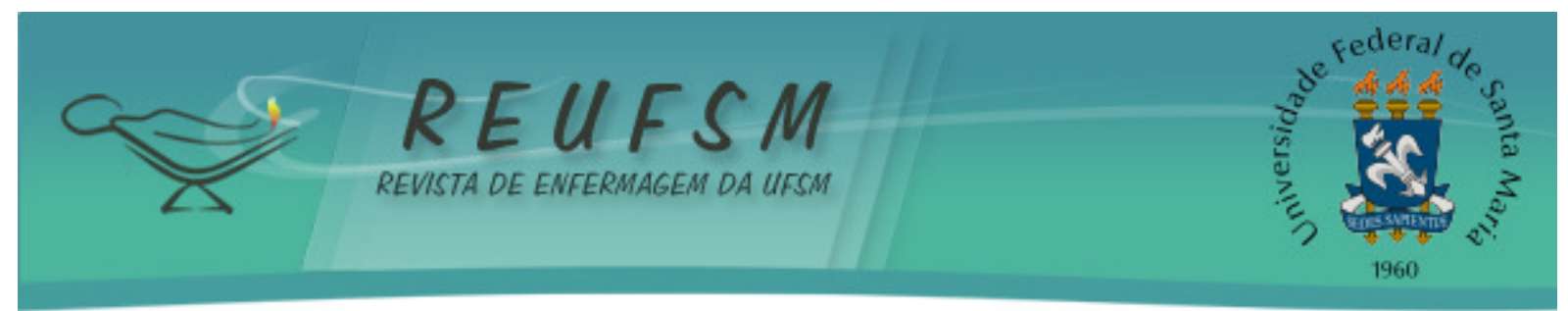

13. Rouquayrol MZ, Almeida Filho N. Epidemiologia e saúde. $6^{\mathrm{a}}$ ed. Rio de Janeiro: MEDSI; 2003. p. 37-79.

14. Brasil. Ministério da Saúde. Secretaria de Vigilância em Saúde. Programa Nacional de DST e Aids. Protocolo para a prevenção de transmissão vertical de HIV e sífilis: manual de bolso. Brasília (DF); 2007.

15. Silva MJM, Mendes WS, Gama MEA, Chein MBC, Veras DS. Perfil clínico-laboratorial de crianças vivendo com HIV/AIDS por transmissão vertical em uma cidade do Nordeste brasileiro. Rev Soc Bras Med Trop [Internet]. 2010 jan/fev [acesso em 2013 maio 16];43(1):32-5. Disponível em: http://www.scielo.br/pdf/rsbmt/v43n1/a08v43n1.pdf.

16. Vieira ACBC, Miranda AE, Vargas PRM, Maciel ELN. Prevalência de HIV em gestantes e transmissão vertical segundo perfil socioeconômico, Vitória, ES. Rev Saúde Pública [Internet]. 2011 jul [acesso em 2013 maio 16];45(4):644-51. Disponível em: http://www.scielo.br/pdf/rsp/v45n4/2487.pdf.

17. Brasil. Ministério da Saúde. Secretaria de Assistência à Saúde. Programa Nacional de DST e AIDS. Aleitamento x mulheres infectadas pelo HIV: recomendações. Brasília (DF); 1995.

18. Brasil. Ministério da Saúde. Secretaria Executiva. Coordenação Nacional de DST e AIDS. Recomendações para profilaxia da transmissão vertical do HIV e terapia anti-retroviral em gestantes. Brasília (DF); 2003. (Manuais; 46).

19. Oliveira MIC, Silva KS, Gomes Junior SC, Fonseca VM. Resultado do teste rápido antiHIV após o parto: uma ameaça à amamentação ao nascimento. Rev Saúde Pública [Internet]. 2010 fev [acesso em 2013 jun 18];44(1):60-9. Disponível em: http://www.scielo.br/pdf/rsp/v44n1/07.pdf.

20. Melo MC, Pimenta AM. Característica epidemiológica da AIDS na população com mais de 50 anos em Betim e microrregião. Rev Enferm Cent-Oeste Min. 2012 set/dez;2(3):419-27.

21. Fernandes RCSC, Ribas GF, Silva DP, Gomes AM, Medina-Acosta E. Desafios operacionais persistentes determinam a não redução da transmissão materno-infantil do HIV. J Pediatr [Internet]. 2010 nov/dez [acesso em 2013 maio 16];86(6). Disponível em: http: //www.scielo.br/pdf/jped/v86n6/v86n6a10.pdf.

22. Lana FCF, Lima AS. Avaliação da prevenção da transmissão vertical do HIV em Belo Horizonte, MG, Brasil. Rev Bras Enferm [Internet]. 2010 jul/ago [acesso em 2013 maio 16];63(4):587-94. Disponível em: http://www.scielo.br/pdf/reben/v63n4/14.pdf.

23. Machado MMT, Galvão MTG, Kerr-Pontes LRS, Cunha AJLA, Leite AJM, Lindsay AC, et al. Acesso e utilização de fórmula infantil e alimentos entre crianças nascidas de mulheres com HIV/AIDS. Rev Eletrônica Enferm [Internet]. 2007 [acesso em 2013 maio 16];9(3):699711. Disponível em: https://www.revistas.ufg.br/index.php/fen/article/view/7477/5297.

24. Trombini ES, Schermann LB. Prevalência e fatores associados à adesão de crianças na terapia antirretroviral em três centros urbanos do sul do Brasil. Ciênc Saúde Coletiva [Internet]. 2010 jan/mar [acesso em 2013 maio 16];15(2):419-25. Disponível em: http://www.scielo.br/pdf/csc/v15n2/v15n2a18.pdf.

25. Nemes MIB, Castanheira ERL, Santa Helena ET, Melchior R, Caraciolo JM, Basso CR, et al. Adesão ao tratamento, acesso e qualidade da assistência em AIDS no Brasil. Rev Assoc Med Bras [Internet]. 2009 [acesso em 2013 maio 16];55(2):207-12. Disponível em: http://www.scielo.br/pdf/ramb/v55n2/28.pdf. 


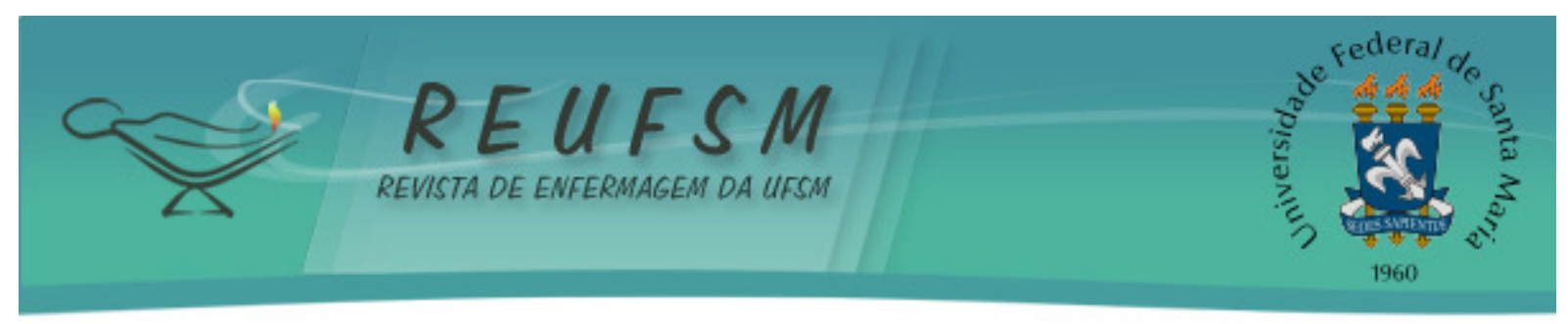

26. Ceccato MG, Acurcio FA, César CC, Bonolo PF, Guimarães MDC. Compreensão da terapia anti-retroviral: uma aplicação de modelo de traço latente. Cad Saúde Pública [Internet]. 2008 jul [acesso em 2013 maio 16];24(7):1689-98. Disponível em: http://www.scielosp.org/pdf/csp/v24n7/23.pdf.

27. Silva EFR, Lewi DS, Vedovato GM, Garcia VRS, Tenore SB, Bassichetto KC. Estado nutricional, clínico e padrão alimentar de pessoas vivendo com HIV/Aids em assistência ambulatorial no município de São Paulo. Rev Bras Epidemiol [Internet]. 2010 dez [acesso em 2013 maio 16];13(4):677-88. Disponível em: http://www.scielo.br/pdf/rbepid/v13n4/12.pdf

Data de recebimento: 16/05/2013

Data de aceite: $29 / 07 / 2013$

Contato com autor responsável: Márcio Cristiano de Melo

Endereço: Rua Tenente Geraldo de Souza Clóvis, 362 ap 203, Angola, Betim-MG.

CEP: $32604-090$

E-mail: enf.marciomelo@gmail.com 